\title{
Core Loss Estimation for an Inverter-fed Induction Motor With More Accurate Realisation of Material Non-linearity and Impact of Hysteresis Minor Loops
}

This paper was downloaded from TechRxiv (https://www.techrxiv.org).

\section{LICENSE}

CC BY-NC-SA 4.0

SUBMISSION DATE / POSTED DATE

$30-12-2020 / 31-12-2020$

\section{CITATION}

Kumar, Rajendra; Kumar, Praveen (2020): Core Loss Estimation for an Inverter-fed Induction Motor With More Accurate Realisation of Material Non-linearity and Impact of Hysteresis Minor Loops. TechRxiv. Preprint. https://doi.org/10.36227/techrxiv.13502226.v1

$\mathrm{DOI}$ 


\title{
Core Loss Estimation for an Inverter-fed Induction Motor With More Accurate Realisation of Material Non-linearity and Impact of Hysteresis Minor Loops
}

\author{
Rajendra Kumar, Praveen Kumar, Senior Member, IEEE
}

\begin{abstract}
The work presents a fast and accurate core loss evaluation method for inverter-fed induction machines. The proposed method takes into account the influence of time variation of magnetic permeability on the eddy current loss. Furthermore, the proposed method presents an accurate approach to precisely evaluate the hysteresis loss. The results obtained from the proposed method are compared with the existing methods for a $1.5 \mathrm{~kW}$ inverter-driven induction motor. The method shows sufficiently high accuracy with reduced complexity and implementation-time for a wide range of inverter switching frequency and motor's loading.
\end{abstract}

Index Terms-Induction motor, core loss, eddy current loss, hysteresis loss, motor efficiency, inverter, pulse-width modulation (PWM) supply, finite element.

\section{INTRODUCTION}

$\mathbf{E}$ Valuation of core-loss (CL) is one of the critical challenges to precisely determine the efficiency of an inverter fed induction motor (IF-IM). Inverter power supply harmonics increase the rate of change of magnetic field densities at different segments of the iron core and, consequently, the net CL. This increment goes up to $35 \%$ compared to the same in a sinusoidally excited motor of the same fundamental frequency[1]. Moreover, applications like electric traction need a wide range of field weakening (a high frequency-operation), which furthermore increases the CL proportion. A precise determination of the $C L$ is hence a prime requirement to design an efficient IF-IM.

One of the accurate techniques to evaluate the CL at the design stage is the finite element analysis (FEA). In FEA, the CL is calculated in the post-processing of the FEA results. Many time and frequency domain models have been proposed for this post-process calculation [2], [3]. These models are modified for improved versions of either the Steinmetz equation or the classical two-term (eddy current loss term and hysteresis loss term) CL model [3], [4]. The modification passes from the addition of excess loss term [5] to making the loss coefficients variables [6]-[11] and improvements in the Jiles-Atherton (JA) model [12], [13]. All these approaches have their tradeoffs in terms of characterization of loss coefficients [11], lack of flexibility [14], computation time [14], limited application domain [15], and complex realization [13]. In a benchmark development, [16], [17] present fast methods by introducing hysteresis and eddy current loss coefficients. However, the formulation is valid as long as the supply voltage is equal to the magnetizing voltage, i.e., high switching frequency and small load. Other concerns with FEA based techniques are the selection of time step and geometry segmentation. Numerical errors due to inadequate time-stepping lead to a high deviation in the results. [18] presents essential computational requirements for these FEM models, avoiding which lead to erroneous core loss results.

There is a need of a comprehensive technique to determine the CL during the design stage, and hence the objectives of this work are:

- Provide a comprehensive analysis of FEA based CL evaluation approaches for a wide range of three operation indices of an IF-IM viz. Switching frequency and, loading of the motor. This encases the entire range of motors power supply dynamics with several control techniques.

- Propose and an accurate and easy to implement CL model, which can be used at the design stage of the motor. The proposed formulation presents a more accurate way to take into account the variation in lamination thickness with time in the existing methods. Alongside, the work presents an easy-to-implement approach for the evaluation of hysteresis loss with good accuracy.

The paper's organisation is as follows: A qualitative analysis of various CL evaluation methods is given in Section II. Section III presents the proposed CL method. The application of the proposed method for an inverter-fed IM is given in Section V. Section VI shows the comparison of the proposed model and the existing CL models with the measurements. The conclusions are drawn in Section VII.

TABLE I

COMPARISON OF METHODS OF CL EVALUATION

\begin{tabular}{llllllll}
\hline \hline & \multicolumn{3}{c}{ SBM } & \multicolumn{2}{c}{ NSBM } \\
& CC & VC & CC & VC & HLM & \multirow{2}{*}{ Hybrid } \\
\hline Accuracy & & & & & & & \\
Low $B_{m}$, low $f$ & $P_{\text {Eddy }}$ & L & H & L-M & H & NA & VH \\
& $P_{\text {Hyss }}$ & M & L & L-M & L & VH & VH \\
High $B_{m}$, High $f$ & $P_{\text {Eddy }}$ & L & M & L-M & M & NA & VH \\
& $P_{\text {Hyss }}$ & L & L & L-M & L & VH & VH \\
\hline Computation time & & S & M & S & M & VH & VH \\
Model development & NA & M & NA & M & H-VH & VH \\
Flexibility & VH & H & VH & H & L-M & L-M \\
Application range & S & M & S-M & M & H-VH & H-VH \\
\hline \hline
\end{tabular}

CC: Constant Coefficients; VC: Variable Coefficients; S: Small; L: Low; M: Medium; H: High; VH: Very High; HLM: Hysteresis Loss Models; NA: Not Applicable.

\section{Cl Evaluation Methods}

This section presents a modified CL model in the review of the limitations of existing CL models. The section comprises 3 subsection. Subsection-A gives an overview of existing CL models. A qualitative analysis of these models is presented in subsection B. 


\section{A. Classification of CL models}

The Steinmetz equation(SE) and the classical two-term model(CTTM) are the two empirical relations for CL evaluation for a sinusoidal magnetic field variation. However,in an electric machine, due to various reasons like stepwise MMF distribution, variation in air-gap permeance due to slots,magnetic saturation, and power supply harmonics, the stator and rotor's flux density deviates from the sinusoid. As a consequence, $\mathrm{CL}$ estimation with these two relations (i.e.SE, and CTTM) is not suitable for a motor and shows erroneous results. Therefore, researchers have proposed various techniques to estimate CL, and they can be broadly classified as:

1) Superposition based CL models(SBM): Superposition based models use the Fourier decomposition of the flux density (B) waveforms, and net core loss is calculated as the sum of CLs of all the harmonics as

$$
P_{C}=\sum_{i=1}^{n}\left(C_{m i} \cdot f_{i}^{\alpha} B_{m i}^{\beta}\right)
$$

where, $C_{m}$, and, are Steinmetz coefficients.

$$
P_{C}=\sum_{i=1}^{n}\left(k_{h i} \cdot f_{i} B_{m i}{ }^{\alpha}+k_{e i} \cdot f_{i}^{2} B_{m i}{ }^{2}\right)
$$

where, $K_{h}, K_{e}$ and, $\alpha$ are frequency $(f)$ and magnetization $(B)$ dependant constants. The FFT decomposition facilitates the application of the original Steinmetz equation as it is. However, applying the superposition for a non-linear routine is the main concern here, particularly for $P_{H y s}$.

2) Non-superposition based CL models(NSBM): These methods evaluate the CL in the time-domain analysis of the instantaneous magnetization quantities, i.e. $B(t)$ and $H(t)$. This time-domain analysis led to several modifications in the (1) and (2) to present an empirical relation. For example, the first such dynamic time-domain model MSE(Modified Steinmetz Equation) presented the CL using a cycle averaged value of the squared rate of change of $B(t)$, i.e., equivalent frequency $\left(f_{e q}\right)$ as

$$
P_{C}=\left(C_{m} \cdot f_{e q}^{\alpha-1} B_{m}^{\beta}\right) f
$$

where,

$$
f_{e q}=\frac{2}{\Delta B^{2} \pi^{2}} \int_{0}^{T}\left[\frac{d B}{d t}\right]^{2} \cdot d t
$$

The above techniques 1) and 2) can be further branched out and a comprehensive overview is given in Fig.1 Also, there exist hybrid methods which determine the $P_{E d d y}$ using (2) and $P_{\text {Hyss }}$ using hysteresis loss models [18].

\section{B. Qualitative analysis}

All the existing models claim to have good accuracy for the range and the material using which they are developed. Hence, the utilization of these models needs a thorough comparative qualitative analysis. Table I narrates their relative comparison for various application parameters. The estimation error with the above models varies depending on the application. In some cases, the error exceeds $80 \%$ of the estimation [10]. One of the main reasons for higher error is that these approaches are based

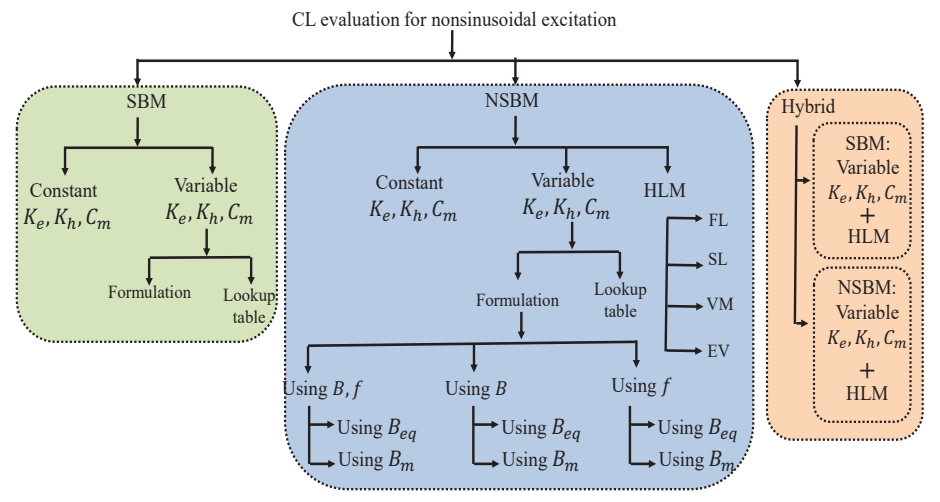

Fig. 1. CL Evaluation techniques in practice. (HLM: Hysteresis loss models viz. FL:Friction like model, $S L$ :Surface loss model, VL:Viscocity based model, $E V$ :Energy based vector model)

on curve fitting an equation to the measurement results, and so the fitting is performed at a certain condition. Hence, the model gives an accurate loss estimation as long as the test conditions match with the conditions in which the data is taken.On the other hand, the mathematical hysteresis models show a much higher accuracy. However, these models require an in-depth insight of the material properties and measurement data under different test conditions. Moreover, the integration of these models into the FEA simulations are not straightforward and simulations take much longer time to run.

From the above discussion, it is evident that there is a need of a comprehensive CL model. Such CL model should address the following difficulties:

- The high error in the estimation of $P_{E d d y}$ due to variation of lamination thickness especially, at higher magnetisation.

- Precise and rapid evaluation of $P_{H y s s}$ for a highly distorted magnetic field variation.

Next section details the proposed CL method addressing the above difficulties.

\section{Proposed CL Method}

The proposed method uses flux density waveforms retrieved from the FEA and evaluates the CL in the post-processing. The formulation of the proposed method employs the superposition based approach for $P_{E d d y}$ and, time-domain approach for $P_{\text {Hyss }}$ [10-12], which, in classical form (for a homogeneous distribution of $B)$ is expressed as [18], [19]

$$
P_{C L_{s}}=\underbrace{\frac{1}{T} \int_{-B_{m}}^{+B_{m}}\left(\frac{\sigma \tau^{2}}{12} \frac{d B(t)}{d t}\right) \cdot d B(t)}_{\mathrm{P}_{\mathrm{Eddy}}} \underbrace{+\frac{1}{T} \oint_{C} H(t) \cdot d B(t)}_{\mathrm{P}_{\mathrm{Hyss}}}
$$

where, $\tau$ is the lamination thickness and $\mu$ is material's magnetic permeability. The dependance of $\tau$ on frequency $(f)$ and material permeability $(\mu)$ due to skin-effect further complicates (5) by replacing $\tau$ with $\tau(f, \mu)$ i.e. [19]

$$
\tau(f, \mu)=\tau \sqrt{3} \pi\left(\frac{\sinh \varepsilon-\sin \varepsilon}{\varepsilon(\cosh \varepsilon-\cos \varepsilon)}\right)^{1 / 2}
$$


where,

$$
\varepsilon=\tau \sqrt{\mu f \sigma \pi}
$$

The firsthand computation of (5) is difficult because it uses $B(t)$ as primary variable and, frequency dependant permeability expression i.e. (6). To simplify (5) and, use it for CL estimation, the following modifications have to be done:

1. Changing of the equation's domain (i.e. from $B(t)$-variable form to time-variable form).

2. Assuming the permeability time-invariant for the range of integral.

In the the following subsections, the use of equation (5) for CL estimation is explained.

\section{A. Eddy current loss $\left(P_{E d d y}\right)$}

Rewriting the integral term of $P_{E d d y_{S}}(5)$ in time-variable form gives

$$
P_{E d}(f, \mu)=\frac{1}{T} \frac{\sigma \tau(f, \mu)^{2}}{12} \int_{0}^{T}\left(\frac{d B(t)}{d t}\right)^{2} \cdot d t
$$

Evaluation of (8) for a sinusoidal field variation $(B(t))$ with time-independent $\tau$ i.e. $\tau(f, \mu)$ provides

$$
P_{E d}\left(f, \mu, B_{\max }\right)=\left(\frac{\sigma \tau(f, \mu)^{2}}{6}\right) B_{\text {max }}^{2} f^{2}
$$

To apply (9) for a nonsinusoidal field variation $(B(t))$, magnitudes of all the harmonic orders are calculated and net $P_{E d d y_{S}}$ is determined as sum of $P_{E d d y_{S}}$ s of all harmonics i.e. for

$$
\begin{gathered}
B(t)=\sum_{i=1}^{n} B_{\max i} \cos \left(\omega_{i} t+\phi_{i}\right) \\
P_{E d d y s}=\sum_{i=1}^{n} P_{E d}\left(f_{i}, \mu_{i}, B_{\max _{-} i}\right)
\end{gathered}
$$

Generally, while solving (8) to get (9), the material permeability $(\mu)$ is assumed constant for a particular value of $B_{\max }$ and $f$. This, in fact simplifies the evaluation of (8) treating $\tau(f, \mu)$ a constant. In [20], this constant $\mu$ is taken corresponding to the $B_{\max }$ whereas, [21] suggests the $\mu$ at average value of excitation.

However, the value of $\mu$ depends upon the magnetisation (as shown in Fig.2-(b)), and hence, it does not remain constant for all the instants of a time period. Fig. 2(c) shows the variation of $\mu$ over a time period i.e., $\mu(B(t))$. Value of $\mu$ is minimum at the maximum value of the excitation. Hence, taking the $\mu$ of maximum magnetisation overestimates the lamination thickness (as per (6)) and consequently gives higher $P_{E d d y_{S}}$ value(as per (9)). Also, it is imperative to mention here that the variation in $\mu$ within the cycle increases with the increase in the maximum magnitude of the waveform $\left(B_{\max }\right)$. The Fig.2(c)-(d) show its variation for both higher and smaller magnetisation $B_{\text {max }}$. As a consequence, $\tau$ also modulates along a fixed value $\tau(f, \mu)$ (as shown in Fig.2(e)) calculated by (6)(7). As mentioned in Section II-C, the variation in $\tau$ has been one of the major sources of error in $P_{E d d y}$ estimation. Hence, the expression (8) needs to be evaluated with taking the timevariation of $\tau$ into account as

$$
P_{E d}\left(f_{i}\right)=\frac{1}{T_{i}} \frac{\sigma}{12} \int_{0}^{T_{i}} \tau\left(f_{i}, \mu(B(t))^{2}\left(\frac{d B(t)}{d t}\right)^{2} d t\right.
$$

In a simplest form, the variation in $\tau$ (as shown in Fig.2-(e)) can be written as

$$
\tau(f, t)=\tau_{0 f}+\Delta \tau_{f}|\sin (\omega t)|
$$

where,

$$
\begin{aligned}
\tau_{0 f} & =\frac{\tau\left(f, \mu_{\max }\right)+\tau\left(f, \mu_{\min }\right)}{2} \\
\Delta \tau_{f} & =\tau\left(f, \mu_{\min }\right)-\tau\left(f, \mu_{\max }\right)
\end{aligned}
$$

Solving the expression (12) and (13) for sinusoidal $B(t)$ gives

$$
P_{E d}\left(f, B_{\max }, \tau^{\prime}\right)=\left(\frac{\sigma \tau^{\prime}(f, \mu)^{2}}{6}\right) B_{\max }^{2} f^{2}
$$

where, $\tau^{\prime}(f, \mu)$ comes out as

$$
\tau^{\prime}(f, \mu)=\left(\tau_{0 f}^{2}+\left(\frac{\Delta \tau_{f}}{4}\right)^{2}\right)^{1 / 2}
$$

which is the root mean squared (rms) value of $\tau(f, t)$. The proposed method hence suggests the evaluation of (12) with taking the rms of the $\tau$. This $r m s$ value of $\tau(f, t)$ is calculated using (6), (14) and,(15). The proposed modification does not impose the restriction on applying the superposition and, the $P_{E d d y_{S}}$ for a non-sinusoidal $B(t)$, with it is calculated as

$$
P_{E d d y s}=\sum_{i=1}^{n} P_{E d}\left(f_{i}, B_{\max _{-} i}, \tau_{i}{ }^{\prime}\right)
$$

where, $P_{E d}\left(f, B_{\max }, \tau^{\prime}\right)$ is calculated using (16). For numerical values of B-data obtained by FEA, the discrete form of the proposed evaluation (18) is used as

$$
P_{E d}\left(f_{i}\right)=\frac{1}{N_{i}} \frac{\sigma}{12} \sum_{k=1}^{N_{i}} \tau^{\prime}\left(f_{i}, \mu(B(k))^{2} \frac{\left(B_{k+1}-B_{k}\right)^{2}}{t_{k+1}-t_{k}}\right.
$$

\section{B. Hysteresis loss $P_{H y s}$}

The term for $P_{H y s_{s}}$ in (5) is $f$-times the area of the hysteresis loop in $B-H$ plane. In inverter fed motors, flux density waveforms (of different motor segments) consist of higherorder time harmonics, which results in several minor loops. Net hysteresis loss in these cases is taken to be the sum of areas of all the loops[11]-[12]. However, this method results in higher $P_{H y s_{s}}$ because the sum of areas of the loops created by each harmonic independently is greater than the actual hysteresis area. The reason for the higher estimation of $P_{H y s_{s}}$ is mainly due to the following three reasons:

- The addition of a harmonic in a sinusoidal B-waveform doesn't always create a minor loop. As shown in Fig.3 ( $a$ and $b$ ), both the B-waveforms (of equal fundamental component $B_{\max _{1}}$ ) comprise equal magnitudes of $3^{\text {rd }}$ and $5^{\text {th }}$ harmonics $\left(B_{\text {max }_{3}}, B_{\text {max }_{5}}\right)$ but their B-H loops are 


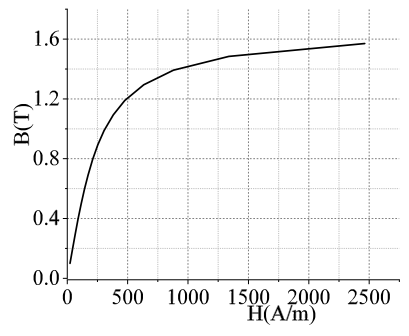

(a)

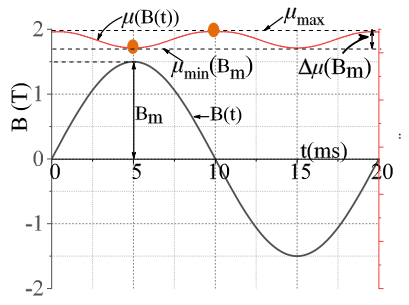

(c)

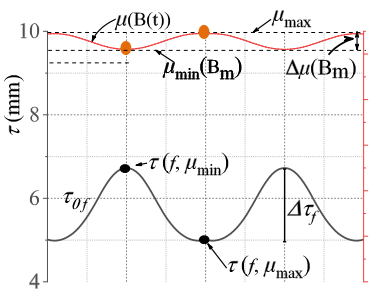

(e)

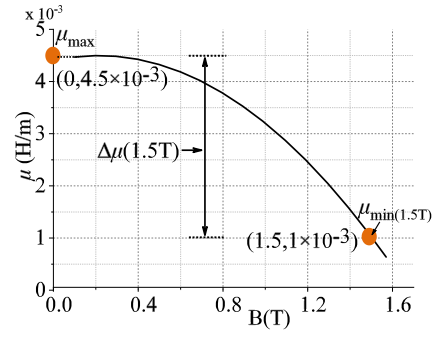

(b)

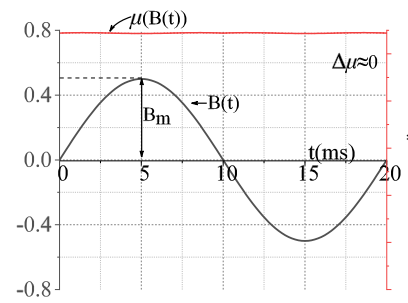

(d)

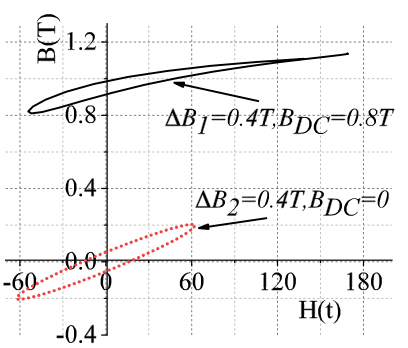

(f)
Fig. 2. (a) B-H curve of the material 50C350; (b) Variation of differential permeability $(\mu)$ with $B(t)$; (c) Variation in $(\mu)$ for sinusoidal excitation of higher $B_{\max }$; (d) Variation in $(\mu)$ for sinusoidal excitation of smaller $B_{\max }$; (e) Variation in $\tau$ for the $\mu$ of (c); (f) The impact of DC-offset on the area of a minor loop.

different.The B-H loop of B-waveform (a) does not form any minor loop whereas, the B-H loop of B-waveform(b) results in two minor loops, each corresponding to the one harmonic component.

- the area of the minor loop (if generated) for a particular harmonic is not exactly equal to the area of the hysteresis loop created by that harmonic independently with the same magnitude.The minor loop area is affected by the DC-bias offered at its location by the major loop. Fig.2(f) shows this impact taking DC offset 0T and, 0.8T.

- it is not necessary that the difference of time instants of maxima and minima of a minor loop provides the time period of a particular harmonic. The superposition of multiple harmonics with different phase-angles may shift the location of these maxima and minima.

The above limitations result in the inaccurate prediction of the hysteresis loss for a distorted B-waveform using an empirical expression. Modelling of the hysteresis loop gives a good solution. The Preisach model, the energetic model, and the Jiles-Atherton model are the prominent hysteresis loop models. These models use $\mathrm{H}$ (or B) as an independent variable and compute the corresponding $\mathrm{B}$ (or $\mathrm{H}$ ) value. HL is created using these $\mathrm{B}-\mathrm{H}$ values for one complete cycle.

The proposed method puts forward a new alternate timedomain approach to determine the area of HL for a distorted

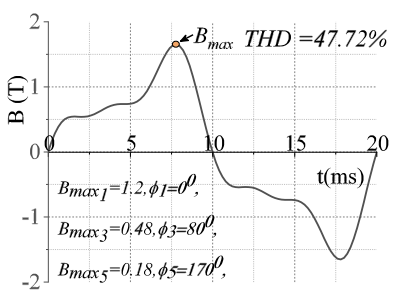

(a)

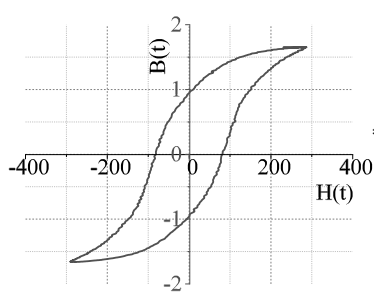

(c)

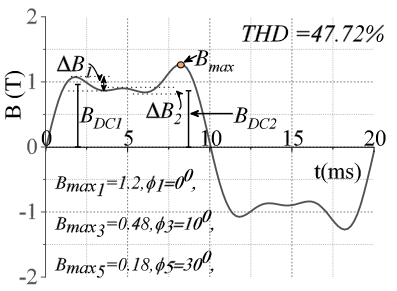

(b)

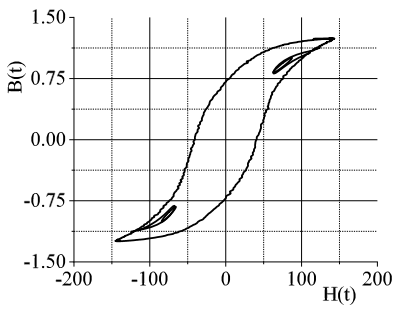

(d)
Fig. 3. (a) Nonsinusoidal waveform-A; (b) Nonsinusoidal waveform-B; (c) Hysteresis loop for waveform-A; (d) Hysteresis loop for waveform-B.

B-waveform without evaluating the $\mathrm{H}$ (i.e. without forming the major and minor HL). The method uses the following two data: a).measured values of HL areas(dynamic) with sinusoidal excitations as a prerequisite which are evaluated using Epstein frame as detailed in section III. b).time-instant B values of the distorted B-waveform which can be directly retrieved from the magnetic vector potential based formulation in FEM. Due to the symmetry of B-waveform, only the half-cycle data is used here. In execution of the proposed method, time spaced half cycle B-data is examined sequentially for its rate of change to get all its local and global extrema $\left(B_{1}, B_{2}, B_{3} . . B_{M}\right.$ as shown in Fig.3-(b)) as points of zero slope. From the sequentially stored values of these extrema, the net HL area is evaluated using the measured HL areas for sinusoidal magnetisation of different magnitudes as described below:

- area of major $\operatorname{loop}\left(A_{m}\right)$ - the maximum absolute value of the stored extrema $\left(B_{\max }\right)$ corresponds to the major loop. Hence, the loop area measured for maximum magnetisation $=B_{\max }$ for sinusoidal excitation is taken as an area of major loop.

- the area of a minor $\operatorname{loop}\left(A_{i}\right)$ - the difference between consecutive maxima and minima $\left(\Delta B_{i}=B_{n}-B_{n-1}\right)$ gives the height of minor loop in B-H plane. The measured HL area corresponding to the maximum $\mathrm{B}$ value equal to the half of the $\Delta B_{i}$ is taken as the area of the minor loop(with zero DC bias)i.e.

$$
A_{0 i}=A_{\text {measured }}\left(\frac{\Delta B_{i}}{2}\right)
$$

where $A_{\text {measured }}\left(B_{i}\right)$ is the measured value of the HL area with the sinusoidal magnetization of the maximum magnitude of $B_{i}$ without DC bias. The DC bias offered at the location of a minor loop is equal to $B_{D C_{i}}=\left(B_{n}+\right.$ $\left.B_{n-1}\right) / 2$ )(Fig.3(b)). The area of minor loop with this DC bias is calculated as [22]

$$
A_{i}=A_{0 i}\left(1+k_{D C} B_{D C_{i}}^{\alpha}\right)
$$


where $k_{D C}$ and $\alpha$ are the constants representing the variation in HL area with DC bias. They are obtained from the measurements as per [22] and, there values for the steel used in this work are 0.67 and 2 respectively.

- total area of the HL and, the corresponding specific hysteresis loss is determined as

$$
P_{H y s_{-} S}=\left(A_{m}+\sum_{i=1}^{L} A_{i}\right) f
$$

where $L$ and $f$ are total number of the minor loops and fundamental frequency respectively.

The proposed method uses hysteresis loss data with sinusoidal excitation as a prerequisite which are evaluated as detailed in Section IV.

\section{CL CHARACTERISATION OF MATERIAL}

The IM used in this work is made of ferromagnetic core material 50C350. The Loss characterisation of this material is done with 352 turns Epstein frame. The setup consists of primary and secondary windings mounted uniformly on the four sides of the frame inside which,the sample ironsheets are placed. A pulsating (of sinusoidal time variation) magnetic field is set up in the sample iron sheets by exciting the primary side from a $3 \mathrm{~kW}$ power amplifier. The THD in voltage (or B-waveform) is limited to $0.5 \%$. The field strength $H(t)$ inside the sample sheet is determined from the primary current as:

$$
H(t)=\frac{N_{P} I(t)}{l_{\text {mean }}}
$$

where, $l_{\text {mean }}$ is mean length of the magnetic flux path, $N_{P}$ is the number of turns in primary winding. The flux density $\mathrm{B}$ is defined via measurement of the voltage induced in the secondary winding $\left(v_{S}(t)\right)$ as

$$
B(t)=\frac{\phi(t)}{A_{\text {core }}}=\frac{1}{A_{\text {core }} N_{S}} \int_{0}^{t} v_{S}(t) d t
$$

where, $A_{\text {Core }}$ is the crosssection area for the flux, $N_{S}$ is the number of secondary windings. The total iron loss for the volume of sample iron-sheets is obtained as shown

$$
P_{\text {Core }}=\frac{N_{P}}{N_{S}}\left(P_{\text {in }}-I^{2} R_{P}\right)
$$

where, $P_{\text {Core }}$ is the total iron loss of the core sample, $P_{I n}$ is the input power for the primary coil, $R_{P}$ is the primary coil resistance.Total core loss estimated with (25) is sum of two losses viz. eddy current loss and hysteresis losses,

$$
P_{\text {Core }}=P_{E d d y}+P_{\text {Hyst }} \text {. }
$$

The components of field strength $H(t)$ in (23) corresponding to these losses are,

$$
H(t)=H(t)_{E d d y}+H(t)_{H y s t} .
$$

The instantaneous value of $H(t)_{E d d y}$ is determined from the instantaneous flux density in (24) as

$$
H(t)_{E d d y}=\frac{\sigma}{12} \tau(f, \mu)^{2} \frac{d B(t)}{d t}
$$

Using (7) and (10) for the effective $\tau(f, \mu)$ lamination thickness, field strength corresponding to the magnetic hysteresis $H(t)_{H y s t}$. is obtained as

$$
H(t)_{H y s t .}=H(t)-\frac{\sigma}{12} t(f, \mu)^{2} \frac{d B(t)}{d t}
$$

The area under the $B(t)$ versus $H(t)$ curve, i.e.,

$$
A_{N e t}=\oint H(t) d B(t)
$$

gives the net core loss $P_{\text {Core }}$ of a cycle, whereas the area under the $B(t)$ versus $H(t)_{H y s t}$. curve

$$
A_{H y s t .}=\oint H(t)_{H y s t .} d B(t)
$$

evaluates the hysteresis loss. Fig.4 shows both these areas for 1.3T, $100 \mathrm{~Hz}$. Fig.5(a)-(d) show the the separated losses $\left(P_{E d d y}\right.$ and $P_{\text {Hyst }}$. $)$ for the range of and magnetisation strength at low and high frequencies.

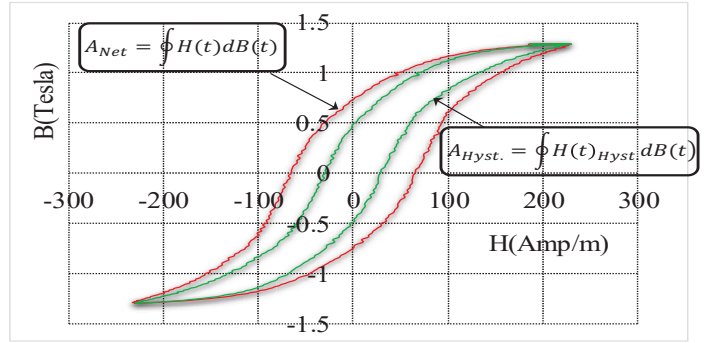

Fig. 4. B-H curves corresponding to the net and magnetising field intensities at $100 \mathrm{~Hz}, 1.3 \mathrm{~T}$.

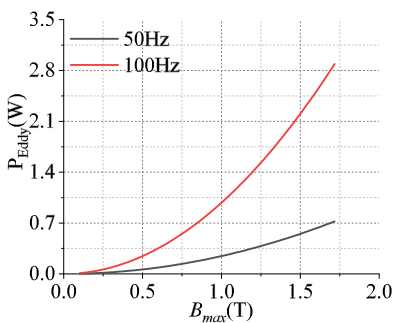

(a)

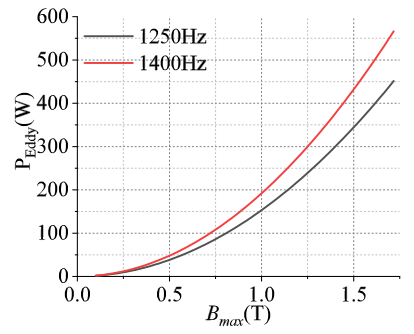

(c)

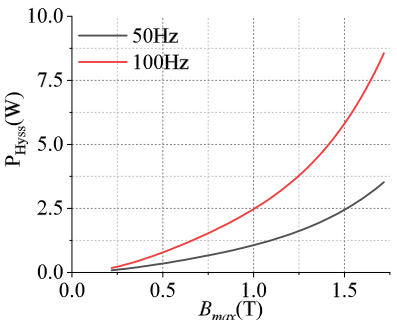

(b)

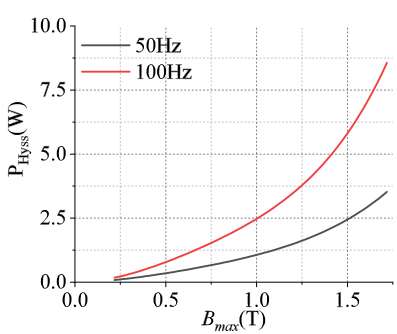

(d)
Fig. 5. CL separation: $P_{E d d y}$ and $P_{H y s t}$. for different frequencies and magnetisations of the rearmost measurements.

\section{CL Evaluation FOR IM}

This section presents the application of the proposed method in the evaluation of CL for an inverter fed IM. For this, a $1.5 \mathrm{~kW}$ three phase IM is selected. Table III shows the 
rated parameters of this IM. As mentioned in Section-IIC, the proposed method uses one cycle time data of flux density, a $2-\mathrm{D}$ FEA model of the $1.5 \mathrm{~kW}$ IM is developed in Maxwell Ansys 19.2. The stator windings of the FEA model are excited with measured current waveforms and, the model is run with the anhysteretic magnetisation and active eddy current effect.

Retrieving the B-data for all the nodes of mesh elements and their CL calculation demands requires storage. Hence, the motor geometry is divided into small segments [2], [23], [25] of homogenous flux density. Both the tangential $\left(B_{T}\right)$ and $\operatorname{radial}\left(B_{R}\right)$ components of magnetic field density inside a segment are constant [2], [23], [25]. Fig.6 shows the motor crosssection with segments. To apply the proposed method,both $B_{T}$ and $B_{T}$ are extracted from the FEA model for one fundamental time period.Using the $B_{T}$ and $B_{T}$, specific CL for the segment evaluated as

a). $P_{E d d y_{S}}$ : using the expression (19) i.e. the evaluation with instantaneous flux density dependant permeability (i.e. $\mu(B(t))$. $P_{E d d y}$ with $B_{T}$ and $B_{R}$ are determined separately and added to get total $P_{E d d y_{S}}$.

b). $P_{H y_{S}}$ : using the expression (22).

Net CL of the machine is estimated as

$$
P_{C L}=\sum_{i=1}^{S} m_{i}\left[P_{H y s_{-} S_{-} i}+P_{E d d y \_S \_}\right]
$$

where, $S$ is the total number of geometry segments and, $m_{i}$ denotes the mass of $i$-th segment.

The FEA model is run for multiple measurements of motor currents to encase the entire range of the IM's operation $(0.5 \mathrm{Nm}$ to $2.5 \mathrm{Nm})$ and carrier frequency variation $(300 \mathrm{~Hz}$ to $5 \mathrm{kHz})$.

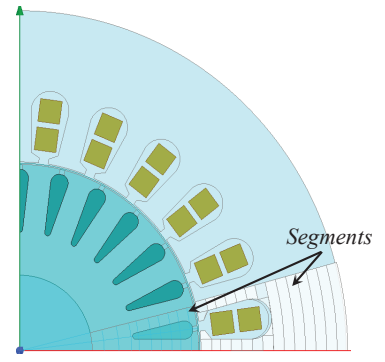

Fig. 6. FEA model of the motor and postprocess geometry segments.

The CL for each operating condition is evaluated applying the proposed method.

\section{EXPERIMENTAL MEASUREMENT OF CL}

Fig.7 shows the results obtained with the proposed technique. For experimental verification of these results, the IM is operated with inverter power supply. CL is evaluated for a total 50 operating points of the motor as given in Table 2. For all these operating points, the speed of the motor is fixed at $2889 \mathrm{rpm}$ by varying its terminal voltage. Fig. 9 shows the voltage variation for the two extremes of switching frequencies i.e. $300 \mathrm{~Hz}$ and $5 \mathrm{kHz}$.
TABLE II

MOTOR OPERATION DOMAIN

\begin{tabular}{lllll}
\hline \hline & $\begin{array}{l}\text { Speed } \\
(\mathrm{rpm})\end{array}$ & $\begin{array}{l}\text { Torque } \\
(\mathrm{Nm})\end{array}$ & $f_{S W}$ & $\begin{array}{l}\text { Power } \\
\text {-supply }\end{array}$ \\
\hline Upper value & 2889 & 2.5 & 5000 & Sine pulse width \\
Lower value & 2889 & 0.5 & 300 & modulation \\
Steps & 1 & 5 & 10 & (SPWM) \\
\hline \hline
\end{tabular}

TABLE III

RATED PARAMETERS OF THE EXPERIMENTAL IM

\begin{tabular}{cccc}
\hline \hline Sr. & Parameter & Unit & Value \\
\hline 1 & Pole-pair & - & 1 \\
2 & $V_{\text {Rated }}$, frequency & Volt./Hz & $415 / 50$ \\
3 & Output power & $\mathrm{kW}$ & 1.5 \\
4 & Core iron-grade & & $50 \mathrm{C} 350$ \\
5 & Lamination thickness & $\mathrm{mm}$ & 0.5 \\
6 & Slots: Stator, Rotor & Number & 36,34 \\
7 & Outer diameter & $\mathrm{mm}$ & 400 \\
8 & Inner diameter & $\mathrm{mm}$ & 400 \\
9 & Stack length & $\mathrm{mm}$ & 400 \\
10 & Air-gap length & $\mathrm{mm}$ & 0.5 \\
\hline \hline
\end{tabular}

\section{A. Measurements-Set-up and procedure:}

Mechanical (Windage and bearing) losses of the IM are first calculated with the sinusoidal power supply as per [24]. Correction for stator resistance for temperature, skin depth and proximity effect is done as[24]:

$$
R_{S}(T, f)=R_{S 0}(1+\alpha(T-20))\left(1+\frac{l_{s}}{l_{w}}\left(k_{n R}(\xi)-1\right)\right.
$$

where, $l_{s}$ is the the stack length and $l_{w}$ is half the mean length of a stator turn. $k_{n R}(\xi)$ is skin and proximity effect factor depends upon the conductor size, number of strands, conductor per slot and slot dimensions[].Stator copper losses are calculated with the measured values of input rms phase currents $\left(I_{S}\right)$ as:

$$
P_{C u S}=R_{S}(T, f) \sum_{i=1}^{3} I_{i}^{2}
$$

The CL is determined using the measured values of the one cycle-averaged input power $\left(P_{I n}\right)$ and then, applying the input-output power balance as:

$$
\begin{gathered}
P_{\text {Core }}=P_{\text {In }}-P_{C u S}-s\left(P_{I n}-P_{C u S}-P_{\text {Core }}\right)-P_{\text {Out }} \\
P_{\text {Core }}=P_{\text {In }}-P_{C u S}-\frac{P_{\text {Out }}}{1-s}
\end{gathered}
$$

where,

$$
P_{\text {Out }}=P_{\text {Shaft }}+P_{\text {Windage }}+P_{\text {Bearing }}
$$

and,

$$
P_{\text {Shaft }}=T_{\text {Shaft }} \omega_{r}
$$

$T_{\text {Shaft }}$ and $\omega_{r}$ are the measured values of shaft torque and rotor speed in $\mathrm{rad} / \mathrm{s}$ respectively using torque sensor (Kistler 4503B) and CoMo Torque Type 4700 data acquisition system. Other equipments of the test-bench are: a) 2-level, H-bridge three-phase inverter b). $1.5 \mathrm{~kW}$ IM motor prototype c). Digital oscilloscope: YOKOGAWA DLM2054 with voltage and cur- 


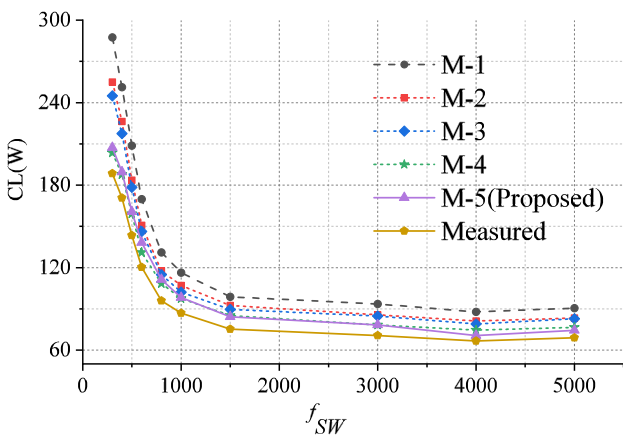

(a)

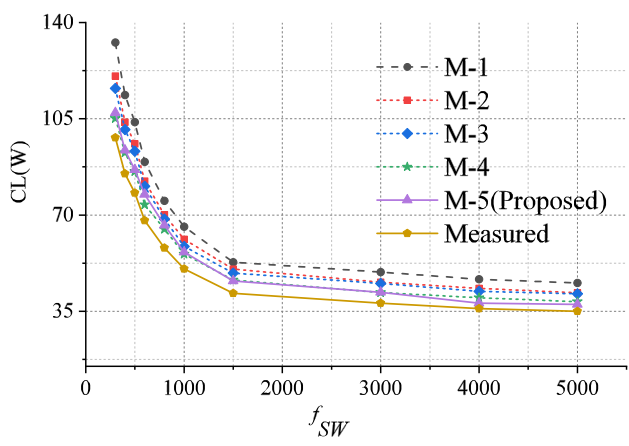

(c)

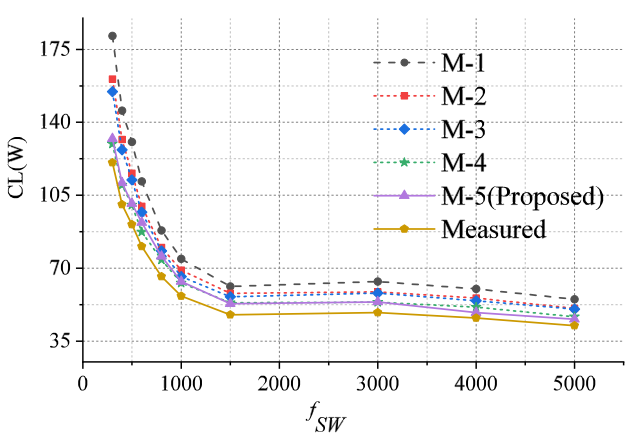

(b)

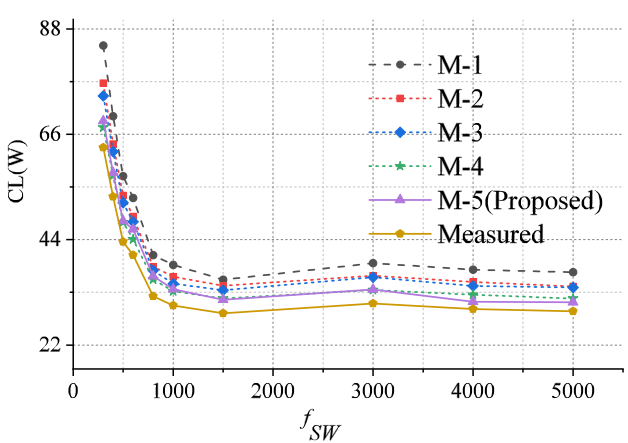

(d)

Fig. 7. Measured and calculated CL values at different $f_{S W}$ for $T_{L}$ equal to (a)2.5 Nm, (b)1.5 Nm(a)1.0 Nm(a)0.5 Nm

rent probes d). Voltage and current transducers: LEM sensors LV25-P and LA 15P f). dSpace DS1103 for data acquisition f).Photo-tachometer. Fig. 8 shows the experimental setup.

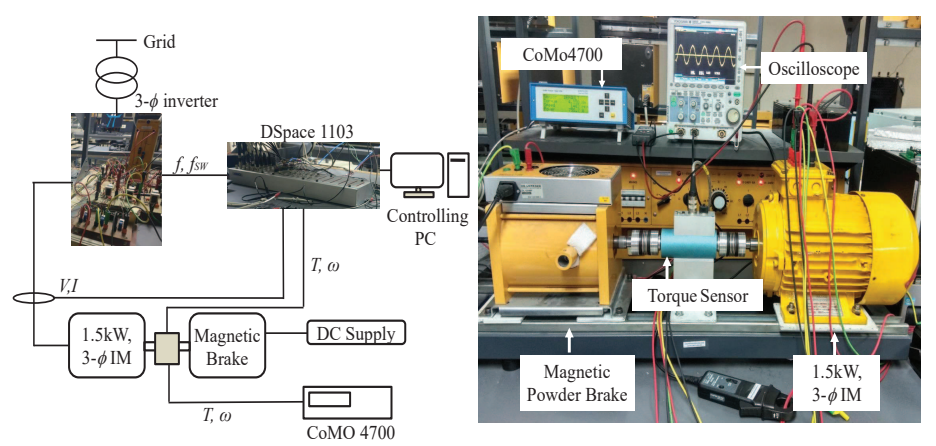

Fig. 8. Experimental setup: Schematic and test-bench

\section{B. Results and discussions:}

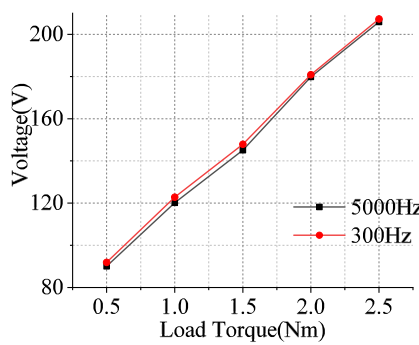

(a)

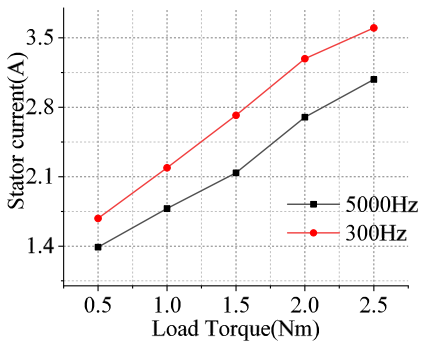

(b)
Fig. 9. Variation of motor's (a) phase voltage and (b) stator current with load torque
The CL for each operating condition is evaluated with the proposed and other widely followed methods of literature. The other methods include:

- CL evaluation with (2) with constant $K_{e}$ and $K_{h}$ values: M-1 $\left(K_{e}=3.717 \mathrm{~W} / \mathrm{Kg} / \mathrm{Hz}^{2} / \mathrm{T}^{2}, K_{h}=0.0449 \mathrm{~W} / \mathrm{Kg} / \mathrm{Hz}^{\alpha} / \mathrm{T}^{\beta}\right)$.

- CL evaluation with (2) with frequency and magnetisation dependant $K_{e}$ (i.e. $K_{e}\left(B_{m}, f_{e q}\right)$ ) and constant $K_{h}$ : M-2.

- CL evaluation with (2) with both $K_{e}$ and $K_{h}$ frequency and magnetisation demandant (i.e.with $\left.K_{e}\left(B_{m}, f_{e q}\right) ; K_{h}\left(B_{m}, f_{e q}\right)\right):$ M-3.

- CL evaluation with using the analytic expression of eddy current loss and, Jiles-Atherton model for the hysteresis loss: M-4.

TABLE IV

ERror Estimates For THE DifFERENT CL METHOdS

\begin{tabular}{cccccc}
\hline \hline Parameter & M-1 & M-2 & M-3 & M-4 & Proposed \\
\hline Max.Error(\%) & 37.5 & 25.03 & 23.11 & 13.25 & 16.21 \\
Min. Error(\%) & 24.43 & 19.68 & 16.34 & 6.62 & 5.11 \\
$\sigma_{\text {Errors }}$ & 8.11 & 5.30 & 4.45 & 1.28 & 1.32 \\
$f_{S W}$ at E(Max) & $300 \mathrm{~Hz}$ & $300 \mathrm{~Hz}$ & $300 \mathrm{~Hz}$ & $300 \mathrm{~Hz}$ & $300 \mathrm{~Hz}$ \\
$f_{S W}$ at E(Min) & $5 \mathrm{Khz}$ & $5 \mathrm{Khz}$ & $5 \mathrm{Khz}$ & $5 \mathrm{Khz}$ & $5 \mathrm{Khz}$ \\
\hline \hline
\end{tabular}

Fig.7 shows the measured and estimated CL values with all the techniques mentioned above. These results draw the following conclusions:

- The proposed method ensures the accuracy of CL estimation at par with method M-4.The maximum and minimum error values with the proposed method are $16.21 \%$ and $5.11 \%$,respectively. Whereas, the same with M-4 are are $13.25 \%$ and $6.62 \%$,respectively. However, the proposed 
method is much easier to implement than M-4 with J-A model.

- For a particular $T_{L}$, both the proposed method and M4 show consistent error (i.e. least values of the standard of deviation of errors $\left(\sigma_{\text {Errors }}\right)$ ). For example, $\sigma_{\text {Errors }}$, at $T_{L}=2 \mathrm{Nm}$, for the proposed method and M-4 are 2.6 are 3.13 respectively, which indicates that the evaluation of hysteresis loop area with the proposed method is close to the same evaluated with JA method. However, there is no direct method available to measure the hysteresis loss (and hence, HL area) independently.

- Method M-2 and M-3 show a higher error at low $f_{S W}$ because time harmonics are dominant, and B-waveforms have larger distortion compared to the same at high $f_{S W}$. The error becomes consistent at higher $f_{S W}$ where, the current waveforms are close to a sinusoid. However, still, the error is high in comparison to the proposed method and $\mathrm{M}-4$.

\section{CONCLUSION}

A fast and accurate iron loss calculation method for IM is proposed for inverter-fed motors in this work. The proposed model determines the eddy current loss by taking the time variation of material permeability into account. Also, the method proposes an easy to implement approach for the evaluation of hysteresis loss. The accuracy of the proposed method is verified by comparing it with the existing CL models. For this, an inverter-fed IM is driven for a wide range of switching frequencies and load-torque. The CL of the IM is obtained for all these operating points applying the four existing CL methods. The evaluation of CL using the proposed method resulted in the least estimation error. The results obtained using the proposed method are close to the same obtained by an analytical appended Jiles-Atherton method for hysteresis loss with a differential of maximum and minimum error equal to $2.96 \%$ and $1.51 \%$, respectively.

\section{ACKNOWLEDGEMENT}

The authors thank to Computer and Communication Center, IIT Guwahati for granting the access of high performance computing platform-Param-ishan to simulate FEA models of IMs.

\section{REFERENCES}

[1] "Energy Management Guide for Selection and Use of Fixed Frequency Medium AC Squirrel-Cage Poly-Phase Induction Motors,"NEMA Standards Publication MG 10-2001.

[2] Z. Gmyrek, A. Boglietti and A. Cavagnino, "Estimation of Iron Losses in Induction Motors: Calculation Method, Results, and Analysis," in IEEE Transactions on Industrial Electronics, vol. 57, no. 1, pp. 161-171, Jan. 2010, doi: 10.1109/TIE.2009.2024095.

[3] H. Domeki et al., "Investigation of benchmark model for estimating iron loss in rotating machine," in IEEE Transactions on Magnetics, vol. 40, no. 2, pp. 794-797, March 2004, doi: 10.1109/TMAG.2004.825442.

[4] C. P. Steinmetz, On the law of hysteresis, Trans. Amer. Inst. Elect. Eng., vol. 9 , no. 1, pp. 164, Jan. 1892.

[5] G. Bertotti, Physical interpretation of eddy current losses in ferromagnetic materials. I. Theoretical considerations, J. Appl. Phys., vol. 57, no. 6, pp. 21102117 , Mar. 1985.
[6] D. M. Ionel, M. Popescu, S. J. Dellinger, T. J. E. Miller, R. J. Heideman and M. I. McGilp, "On the variation with flux and frequency of the core loss coefficients in electrical machines," in IEEE Transactions on Industry Applications, vol. 42, no. 3, pp. 658-667, May-June 2006, doi: 10.1109/TIA.2006.872941.

[7] D. M. Ionel, M. Popescu, M. I. McGilp, T. J. E. Miller, S. J. Dellinger and R. J. Heideman, "Computation of Core Losses in Electrical Machines Using Improved Models for Laminated Steel," in IEEE Transactions on Industry Applications, vol. 43, no. 6, pp. 1554-1564, Nov.-dec. 2007, doi: 10.1109/TIA.2007.908159.

[8] M. Popescu, D. M. Ionel, A. Boglietti, A. Cavagnino, C. Cossar and M. I. McGilp, "A General Model for Estimating the Laminated Steel Losses Under PWM Voltage Supply," in IEEE Transactions on Industry Applications, vol. 46, no. 4, pp. 1389-1396, July-Aug. 2010, doi: 10.1109/TIA.2010.2049810.

[9] Zhao Haisen, , Zhang Dongdong, Wang Yilong, Zhan Yang, and Xu Guorui. "Piecewise variable parameter loss model of laminated steel and its application in fine analysis of iron loss of inverter-fed induction motors.” . In 2016 IEEE Industry Applications Society Annual Meeting (pp. 1-8).2016.

[10] R. Roy, K. K. Prabhakar, and P. Kumar, "Core-loss calculation in different parts of induction motor," IET Electric Power Appl., vol. 11, no. 9, pp. 1664-1674, 2017.

[11] W. Tong, , S. Li, R. Sun, L. Sun, and R. Tang. "Modified Core Loss Calculation for High-Speed PMSMs with Amorphous Metal Stator Cores".IEEE Transactions on Energy Conversion (2020): 1-1.

[12] W. Li, , I. H. Kim, S. M. Jang, and C. S. Koh. "Hysteresis Modeling for Electrical Steel Sheets Using Improved Vector Jiles-Atherton Hysteresis Model".IEEE Transactions on Magnetics 47, no.10 (2011): 3821-3824.

[13] K. Hoffmann, , J. P. A. Bastos, J. V. Leite, N. Sadowski, and F. Barbosa. "A Vector JilesAtherton Model for Improving the FEM Convergence".IEEE Transactions on Magnetics 53, no.6 (2017): 1-4.

[14] A. Krings and J. Soulard, Overview and Comparison of Iron Loss Models for Electrical Machines, Journal of Electrical Engineering, vol. 10 , no. 3, pp. 162169,2010 .

[15] D. Zhang, H. Dai, H. Zhao and T. Wu, "A Fast Identification Method for Rotor Flux Density Harmonics and Resulting Rotor Iron Losses of Inverter-Fed Induction Motors," in IEEE Transactions on Industrial Electronics, vol. 65, no. 7, pp. 5384-5394, July 2018, doi: 10.1109/TIE.2017.2779432.

[16] A. Boglietti, , A. Cavagnino, and M. Lazzari. "Fast Method for the Iron Loss Prediction in Inverter-Fed Induction Motors".IEEE Transactions on Industry Applications 46, no.2 (2010): 806-811.

[17] A. Boglietti, , A. Cavagnino, D. M. Ionel, M. Popescu, D. A. Staton, and S. Vaschetto. "A General Model to Predict the Iron Losses in PWM Inverter-Fed Induction Motors".IEEE Transactions on Industry Applications 46, no.5 (2010): 1882-1890.

[18] L. R. Dupre, R. V. Keer, and J. A. A.Melkebeek, On a magnetodynamic model for the iron losses in non-oriented steel laminations, Journal of Physics D: Applied Physics, vol. 29, no. 3, pp. 855861, 1996.

[19] J. Szczygowski, , and P. Jaboski. "Computation of core losses in electrical sheets used in electrical machines".IET Electric Power Applications 14, no.2 (2020): 291-296

[20] A. M. Takbash, , and P. Pillay. "A modified analytical method for core losses calculation in magnetic laminations for a wide range of frequency and flux density." . In 2015 IEEE International Electric Machines Drives Conference (IEMDC) (pp. 1109-1114).2015.

[21] M. Ibrahim and P. Pillay, "Core loss prediction in electrical machine laminations considering skin effect and minor hysteresis loops," 2012 IEEE Energy Conversion Congress and Exposition (ECCE), Raleigh, NC, 2012, pp. 2681-2687, doi: 10.1109/ECCE.2012.6342536.

[22] S. Xue, , J. Feng, S. Guo, Z. Chen, J. Peng, W. Q. Chu, L. R. Huang, and Z. Q. Zhu. "Iron Loss Model Under DC Bias Flux Density Considering Temperature Influence".IEEE Transactions on Magnetics 53, no.11 (2017): 1-4.

[23] "R. Roy, K. K. Prabhakar and P. Kumar, "Core-loss calculation in different parts of induction motor," in IET Electric Power Applications, vol. 11, no. 9, pp. 1664-1674, 11 2017, doi: 10.1049/iet-epa.2017.0369.

[24] "Rotating electrical machines - part 2-1:standard methods for determining losses and efficiency from tests (excluding machines for traction vehicles)," IEC Std 60034-2-1, Sep. 2007.

[25] "B. Tekgun, Y. Sozer, I. Tsukerman, P. Upadhyay and S. Englebretson, "Core Loss Estimation in Electric Machines With Flux-Controlled Core Loss Tester," in IEEE Transactions on Industry Applications, vol. 55, no. 2, pp. 1299-1308, March-April 2019, doi: 10.1109/TIA.2018.2874352. 